\title{
Impacto de la gestión de calidad en la estructura organizativa y en la innovación de la industria azucarera colombiana
}

\author{
MERCEDES FAJARDO ORTIZ, Ph.D.(c) $)^{1,2,3}$ \\ Profesora tiempo completo, Universidad Icesi, Colombia. \\ mfajardo@icesi.edu.co \\ LINA MARCELA CANO VELÁSCO \\ Universidad Icesi, Colombia. \\ marcelite@hotmail.com \\ MONICA CECILIA TOBÓN GORDILLO \\ Universidad Icesi, Colombia. \\ monicatobon09@gmail.com
}

\section{RESUMEN}

El estudio analiza el posible impacto de la gestión de calidad en la estructura organizativa y en la innovación en tres ingenios azucareros colombianos. Se creó un instrumento para recolectar información que fue diligenciado por algunos colaboradores. Los resultados indican que los ingenios presentan estructuras organizativas formalizadas y una menor especialización horizontal, haciendo énfasis en la formación del personal. No se podría afirmar que implementar un sistema de gestión de calidad genera cambios en la estructura, los niveles jerárquicos, descentralización vertical y en los dispositivos de enlace laterales. Tampoco se evidencia una diferencia significativa en la innovación de los tres ingenios. Futuras investigaciones en el tema podrían ampliarse a otros ingenios del país.

Palabras clave. Estructura organizativa; sistema de gestión de calidad; innovación; industria azucarera colombiana.

Recibido: 30-jun-11, corregido: 30-abr-12 y aceptado: 26-jun-12

Clasificación JEL: M10

@

\footnotetext{
1 Este documento fue seleccionado en la convocatoria para enviar artículos, Call for Papers, realizada en el marco del “II Simposio Iberoamericano de Estudios Gerenciales: Una mirada interdisciplinar a la innovación”, organizado por la revista académica Estudios Gerenciales bajo la dirección de la Facultad de Ciencias Administrativas y Económicas de la Universidad Icesi; el evento tuvo lugar los días 12, 13 y 14 de octubre de 2011, en la ciudad de Cali (Colombia). Este documento fue presentado en las sesiones simultáneas del área de "Innovación en procesos".

2 Este artículo es uno de los resultados del proyecto "Calidad y Diseño Organizativo" financiado por la Universidad Icesi (Colombia). Las autoras agradecen a los profesores del seminario de Gestión de la Calidad y Diseño Organizativo del Doctorado en Dirección de Empresas: Estrategia y Organización de la Universidad de Valencia, España, doctores Teresa Canet y Francisco Balbastre, por sus valiosos aportes y seguimiento en la elaboración de este artículo como trabajo final del seminario en mención. Igualmente, a los pares anónimos de Estudios Gerenciales por sus valiosas sugerencias. 3 Autor para correspondencia. Dirigir correspondencia a: Universidad Icesi, Calle 18 No. 122-135, Pance, Cali, Colombia.
} 
The impact of quality management systems on organizational structure and innovation in the Colombian sugar industry

This study analyzes the potential impact of quality management on organizational structure and innovation in three sugar mills in Colombia. To gather information, a survey was designed and administered to some collaborators. The findings point out that the sugar mills have formal organizational structures in place and a lesser degree of horizontal specialization with a strong focus on personnel training. It is irrefutable that implementing a quality management system necessarily leads to changes in the structure, hierarchical levels, vertical decentralization, and lateral liaison devices. There is also no evidence of significant differences in innovation at any of the three sugar mills. It would be advisable to conduct further research on this topic at other mills in the country in the future.

Keywords. Organizational structure; quality management system; innovation, Colombian sugar industry.

\section{$\infty$}

\section{Impacto da gestão da qualidade na estrutura organizacional e inovação da indústria açucareira colombiano}

O estudo analisa o impacto potencial de gestão da qualidade na estrutura organizacional e inovação em três usinas de açúcar da Colômbia. Foi criado um instrumento para coletar informações que foi empregado por alguns colegas. Os resultados indicam que as usinas apresentam estruturas organizacionais formalizadas e uma menor especialização horizontal, com foco na formação de pessoal. Ninguém pode argumentar que a implementação de um sistema de gestão da qualidade gera mudanças na estrutura, nos níveis de hierarquias, na descentralização vertical e nos dispositivos de conexão laterais. Também não é uma evidência de uma diferença significativa na inovação das três fábricas. Futuras pesquisas sobre o tema poderiam ser estendidas para outras fábricas no país.

Palavras-chave. Estrutura organizacional; sistema de gestão da qualidade; inovação; indústria açucareira colombiana. 


\section{Introducción}

El propósito primordial de este estudio es analizar el posible impacto que la implementación de un sistema de gestión de calidad puede generar en la estructura organizativa, así como en los procesos de innovación de tres ingenios azucareros colombianos. Teniendo en cuenta que dicha implementación modifica de cierta manera la estrategia, en este artículo se considerará la estrategia como la determinación de las metas y objetivos básicos a largo plazo de una empresa, y la adopción de cursos de acción y asignación de los recursos necesarios para lograr dichas metas (Chandler, 1962). Incluye, por tanto, la doble vertiente de medio o instrumento y de fin u objetivo.

La relación entre estrategia y estructura es una de los principales aportes de Chandler (1962) y la modificación de dicha estrategia suele obedecer a cambios externos, lo que a su vez produce cambios en la estructura. Así mismo, la administración de la calidad es un esfuerzo continuo y constante de todas las funciones de una organización para encontrar nuevas maneras de mejorar la calidad de sus bienes y servicios (Deming, 1989). Los procesos exitosos de la gestión de la calidad demandan de las organizaciones cambios profundos en su estructura organizativa (Grant et al., 1994; citado por Bayo \& Merino, 2003).

Existen pocos trabajos que analicen la vinculación entre la implantación del sistema de gestión de calidad, el diseño organizativo y la innovación. Por lo tanto, el objetivo de este artículo es plantear una serie de hipótesis que relacionan la adopción de un sistema de gestión de calidad con varios de los parámetros de diseño organizativo, y estos a su vez, con el análisis comparativo de las condiciones de la organización para incrementar su potencial de innovación. Para lograr este objetivo, se aplicó un instrumento entre diferentes cola- boradores del sector azucarero colombiano, lo que permitió determinar las diferencias entre los ingenios pertenecientes a la muestra.

Así mismo, se supone que las organizaciones que apuestan por el sistema de gestión de calidad tenderán hacia estructuras organizativas más flexibles y participativas, lo que propicia el incremento de su potencial de innovación. Este trabajo es por tanto una aportación novedosa al conocimiento de los procesos de implementación de la gestión de la calidad y del potencial de innovación en las organizaciones colombianas del sector azucarero.

El documento se estructura de la siguiente manera. En primer lugar, se desarrolla el marco referencial del estudio, en donde se presentan los planteamientos teóricos de forma general que permite la formulación de las hipótesis del mismo; seguidamente, se presenta la unidad de análisis, la estructura y la metodología que se plantea utilizar en el desarrollo de este proyecto; finalmente, luego del análisis de resultados, se discuten las conclusiones más significativas.

\section{Antecedentes}

La industria azucarera colombiana está compuesta por aproximadamente 1.200 proveedores de caña de azúcar, con 200.000 hectáreas sembradas y transformadas por trece ingenios. Estos, a su vez, se convierten en proveedores de insumos importantes a la industria papelera, de alimentos y energética en el país. Debido a ello, se han creado instituciones como el Centro de Investigación de la Caña de Azúcar de Colombia (Cenicaña), la Asociación de Cultivadores de Caña de Azúcar de Colombia (Asocaña) y la Asociación Colombiana de Técnicos de la Caña de Azúcar (Tecnicaña); las cuales trabajan con el fin de lograr que el sector sea cada día más eficiente y productivo (Asocaña, 2011). 
Los ingenios azucareros en Colombia nacen en 1864 con el Ingenio Manuelita; siendo este pionero a nivel nacional en azúcar refinada y el primero en obtener la certificación de proveedor Tipo A por parte de la embotelladora Coca-Cola; lo cual le permitió tener acceso a nuevos mercados (Grupo Manuelita, 2011).

Constantemente, la industria azucarera colombiana ha realizado avances tecnológicos para la cosecha, desarrollo para la exportación, integración a negocios complementarios e internacionalización y un compromiso importante con la sostenibilidad del medio ambiente y el mejoramiento de la calidad de vida de los colaboradores (Asocaña, 2011).

Para este estudio se tomó como base el de Bayo \& Merino (2003) que tuvo como objetivo analizar la repercusión que la adopción que los principios y técnicas de la gestión de la calidad total por parte de las organizaciones tiene sobre el diseño de las estructura. La información se obtuvo de una muestra de 965 plantas industriales españolas con más de cincuenta trabajadores pertenecientes a los distintos sectores manufactureros, lo cual permitió contrarrestar una serie de hipótesis previamente argumentadas en la teoría de Mintzberg (1984) sobre el diseño de la estructura organizativa.

Los resultados de esta investigación española reflejaron que las organizaciones que adoptan la gestión de la calidad total presentan una estructura organizativa con una menor especialización horizontal, son más formalizadas, hacen más énfasis en la preparación, utilizan más grupos de mejora y están descentralizadas verticalmente. Así mismo, no corroboraron las hipótesis planteadas en relación a una menor especialización vertical, un mayor adoctrinamiento y una estructura más horizontal en aquellas organizacionales que adoptaron la gestión de la calidad total.
$\mathrm{El}$ anterior estudio se convierte entonces en la mejor herramienta para tratar de extrapolar la investigación, ya que no se encontraron más estudios que pudieran ajustarse al propósito del aquí presentado y, sobre todo, que permitiera analizar las condiciones de la organización para incrementar su potencial de innovación en un sector económico de tanta importancia para Colombia como lo es el caso del azucarero.

El objetivo de este artículo es analizar el impacto que ha tenido la implementación de un sistema de gestión de calidad en el diseño organizativo y en la innovación de tres ingenios del sector azucarero colombiano. Como objetivos específicos se tiene examinar la repercusión que la implementación del sistema de gestión de calidad ha tenido en el diseño de puestos y la superestructura de las tres organizaciones; examinar la repercusión que la implementación del sistema de gestión de calidad ha tenido sobre el diseño de enlaces laterales; examinar la repercusión que la implementación del sistema de gestión de calidad ha tenido sobre el diseño del sistema decisor; y examinar si la repercusión que la implementación del sistema de gestión de calidad y su impacto en el diseño de la estructura, favorece las condiciones de la organización y presentan un incremento en su potencial de innovación.

\section{Marco teórico}

\subsection{El diseño de la estructura de las organi- zaciones y la estrategia}

La organización formal es un producto diseñado según ciertos principios y conocimientos científicos, de conformidad con determinados valores sociales. Igualmente, la organización formal tiene como objetivo producir determinados resultados conscientemente concebidos y perseguidos por el ser humano (Rico \& Fernández, 2002). Según algunos autores: 
Andrews (1999; citado por Mintzberg, Quinn \& Voyer, 1997) define "La estrategia representa un patrón de objetivos, propósitos o metas, así como las políticas y los planes principales para alcanzar estas metas, presentándolos de tal manera que permiten definir la actividad a la que se dedica la empresa, o a la cual se dedicará, así como el tipo de empresa que es o será” (p. 2), Quinn lo amplía a un plan que integra objetivos y políticas que puede ser visto desde cinco perspectivas diferenciadas: un plan de acción a seguir, un patrón de acción consistente en el tiempo, una maniobra para confundir, una posición en un mercado y una forma particular del quehacer de la organización (Mintzberg \& Lampel, 1999), todas ellas coexisten en un diseño organizacional. Para Chandler (1962) la estrategia es la determinación de las metas y objetivos básicos a largo plazo de una empresa, la adopción de cursos de acción y asignación de los recursos necesarios para lograr dichas metas, incluye por tanto, la doble vertiente de medio o instrumento y de fin u objetivo.

Según Mintzberg \& Lampel (1999) la perspectiva original de Selznick, seguido de Chandler y teniendo en cuenta la definición de Andrews, muestra la formación de la estrategia como una forma de lograr el ajuste esencial entre las fortalezas y debilidades internas y las oportunidades y amenazas externas. El término estrategia tiene comúnmente connotaciones clásicas, constreñidas al ámbito del arte militar. Con este término se hace referencia al plan de campaña, teniendo en cuenta diversos factores y aspectos de la situación concernientes a las condiciones del territorio, del adversario y de las propias fuerzas (Menguzzato \& Renau, 1992). Por medio de su estrategia, una organización busca utilizar y desarrollar competencias para lograr una ventaja competitiva, e incrementar su participación en los recursos escasos que pueda obtener de su ambiente.

La estructura de la organización es una consecuencia de la estrategia, de modo que primero se adopta una estrategia y luego se elige un arreglo organizacional. La estructura se diseña para administrar las actividades que surgen de las distintas estrategias adoptadas, lo cual implica la existencia de un orden de jerarquía, cierta distribución y asignación de trabajo, líneas de autoridad y comunicación, y datos e información que fluyen a través de las distintas líneas (Chandler, 1962).

El diseño organizacional es el proceso por medio del cual los gerentes seleccionan y administran los aspectos de estructura y cultura para que una organización pueda controlar las actividades necesarias con las que logra sus metas (Jones \& Ruíz, 2008). Dicho esto, diseñar organizaciones es la actividad por la cual se concibe, construye o se cambia la estructura de una organización con el ánimo de lograr sus objetivos (Mintzberg, 1991; Robbins, 1990; Simon, 1981; Starbuck \& Nystrom, 1981; citados por Rico \& Fernández, (2002).

La relación entre estrategia y estructura es una de los principales aportes de Chandler (1962). La modificación de la estrategia suele obedecer a cambios externos y es lo que produce los cambios en la estructura; concretamente, las transformaciones de crecimiento en las empresas las identifica como expansión geográfica, integración vertical (de servicios realizados por otras empresas, por ejemplo), o diversificación (introduciendo nuevos productos).

Estos cambios son el ambiente de una organización, el cual es la compleja red de fuerzas cambiantes que afectan la manera en que ésta opera así como su acceso a recursos escasos; el ambiente es una contingencia importante para la cual una organización debe planear y adaptarse (Jones \& Ruíz, 2008). 
A medida que se incrementa la complejidad y la incertidumbre en el entorno, las organizaciones adaptan su estructura organizacional a esta contingencia. Van de Ven \& Drazin (1985) explican las formas alternativas de ajuste en la teoría de contingencias; en esta, estos autores examinaron el ajuste según los conceptos de selección, interacción y acercamiento sistémico en la estructura organizacional y, junto con Lawrence, Lorsh y Mintzberg (citados por Pinto, Soto, Gutiérrez \& Castillo, 2003) dieron apertura a una nueva concepción de organización según la cual, la estructura de una organización y su funcionamiento dependen de la interfaz con el ambiente externo.

La teoría de las contingencias adopta los enunciados conceptuales y metodológicos de la teoría general de sistemas, en donde utiliza buena parte de su batería conceptual: entorno, sistema, subsistemas, entradas, salidas, homeostasis, entropía, retroalimentación, etc. El objetivo fundamental de la teoría de las contingencias es la explicación de las características internas de las organizaciones en función de la situación del entorno.

La organización, o sistema total, puede ser entendida en términos del conjunto de sistemas que, a su vez, pueden subdividirse en otros sistemas. Generalmente las organizaciones se inclinan por tener una estructura más horizontal que fomente la comunicación transfuncional y la colaboración; y realizan los ajustes pertinentes entre la estructura interna y el entorno para administrar y controlar más eficientemente disminuyendo igualmente el nivel de incertidumbre (Daft, 2007).

Así mismo, como resultado del entorno cambiante dado por la competencia global, los cambios en la tecnología, las comunicaciones y el transporte, se han generado nuevas oportunidades para las organizaciones de todos los tamaños y en todas las industrias.
Por este motivo, éstas se ven obligadas a cambiar, a revisar nuevamente su forma de realizar negocios y a adaptar sus estructuras. Tales contingencias externas pueden considerarse oportunidades, imperativos o restricciones que influyen en la estructura y los procesos internos de la organización (Pinto et al., 2003).

Ya sea que una empresa compita a través de una estrategia de liderazgo en costo o de una diferenciación de producto, los temas de calidad impregnan cada aspecto de las operaciones (Blocher, Stout, Cokins \& Chen, 2008). Ambas estrategias implican cambios organizacionales y sus objetivos son encontrar nuevas y mejores formas de utilizar sus recursos y capacidades para aumentar la capacidad de crear valor y mejorar los rendimientos de las partes interesadas (Beer \& Walton, 1987).

\subsection{El diseño de la estructura de las organi-} zaciones y la gestión de la calidad

La teoría del sistema socio-técnico fue una de las primeras teorías que propusieron la importancia de cambiar las relaciones de funciones y tareas o las técnicas para aumentar la efectividad organizacional. Trist et al. (citados por Jones \& Ruíz, 2008) argumentan que los gerentes necesitan adaptar u optimizar conjuntamente los sistemas técnico y social de una organización para promover su efectividad. Un sistema que se base en equipos, promoverá el desarrollo de valores y normas que estimularán la eficiencia y la calidad de los productos (Jones \& Ruíz, 2008).

La administración de la calidad es un esfuerzo continuo y constante de todas las funciones de una organización para encontrar nuevas maneras de mejorar la calidad de sus bienes y servicios (Deming, 1989), a la vez que es el esfuerzo persistente y continuo de todos en la empresa por comprender, cumplir y exceder las expectativas del cli- 
ente (MQI-IMA, 1993; citado por Blocher et al., 2008).

Por su parte, aunque cada organización desarrolla su propio enfoque hacia la administración, estas mantienen objetivos comunes como son la satisfacción del cliente, la mejora continua, comprometer en su totalidad a la fuerza laboral, el trabajo en equipo, respaldar en forma activa e incluir a la administración superior, utilizar mediciones no ambiguas y objetivas, reconocer los logros de calidad en una forma oportuna, y proporcionar capacitación de manera continua acerca de la administración de los sistemas de gestión de calidad.

Los objetivos de la administración de la calidad y la continua mejora en la calidad del producto, se basan en los principios arraigados en la teoría del sistema socio-técnico. Una vez que una organización lo adopta, genera un cambio continuo e incremental y se espera que todas las funciones cooperen entre sí para mejorar la calidad, motivando cambios en las relaciones interfuncionales (tareas, funciones y grupos), aumentando la calidad y disminuyendo los costos (Jones \& Ruíz, 2008).

Los procesos exitosos de la gestión de la calidad demandan cambios profundos en la estructura organizativa de las empresas (Grant et al., 1994; citado por Bayo \& Merino, 2003). En este sentido, el modelo de mando y control, da paso a un modelo de consejo y apoyo, el cual afecta las relaciones entre funciones e incluso divisiones, y sólo se convierte en un proceso evolutivo cuando se transforma en una forma de vida de la organización.

De igual forma, estudios sobre el individuo y los grupos muestran que los elementos conductuales de las operaciones grupales deben estar más estrechamente integrados con el diseño de la estructura organizativa, la dotación de personal, la planeación y el control (Koontz; traducido por Ríos, 2000).

El flujo de información y la documentación es una buena manera de verificar la forma en que los procesos funcionan realmente y, solo si contribuye a un crecimiento real en conocimiento de la organización formal y accesible, apoyan el trabajo diario y las acciones de mejora continua.

\subsection{Implicaciones que la adopción del sistema de gestión de calidad tiene sobre el diseño de las organizaciones}

Es importante estudiar las consecuencias del diseño organizacional (Andersen, 2002) y, en este caso, las implicaciones que la adopción del sistema de gestión de calidad tiene sobre el diseño de las organizaciones. Para tal fin, en este estudio se contempla el diseño desde el marco conceptual definido por Mintzberg (1984), basado en la definición de los parámetros de diseño organizativo, el cual permite analizar esta relación y plantear una serie de hipótesis que se contrastarán posteriormente.

Posteriormente se toma como base el proyecto: "El sistema de gestión de calidad sistema de gestión de calidad y el diseño de la estructura organizativa: un estudio empírico" de Bayo \& Merino (2003) (ver Tabla 1). Los parámetros de diseño de las organizaciones, en este caso los ingenios, se pueden recoger en cuatro grupos constituyentes del objeto del diseño con los parámetros asociados a cada uno (Mintzberg, 1984).

Para este trabajo se plantearán y contrastarán hipótesis relacionadas con siete de los nueve parámetros de diseño organizativo, así:

\section{Diseño del puesto}

Hipótesis 1 (H1). Las organizaciones que adoptan el sistema de gestión de calidad presentan una menor especialización horizontal. 
Tabla 1. Parámetros de diseño organizativo|

\begin{tabular}{|c|c|c|}
\hline $\begin{array}{l}\text { Objeto de } \\
\text { estudio }\end{array}$ & Incluye & Parámetro de diseño \\
\hline \multirow{3}{*}{$\begin{array}{l}\text { 1.Diseño del } \\
\text { puesto }\end{array}$} & \multirow[t]{3}{*}{ Cuáles son las tareas y quienes las realizan. } & Especialización \\
\hline & & Formalización \\
\hline & & $\begin{array}{l}\text { Preparación y adoctrina- } \\
\text { miento }\end{array}$ \\
\hline \multirow{3}{*}{$\begin{array}{l}\text { 2.Diseño de la } \\
\text { superestruc- } \\
\text { tura }\end{array}$} & \multirow{3}{*}{$\begin{array}{l}\text { Se plantea como se agrupan los puestos en unidades } \\
\text { y que dimensiones debe tener cada unidad. Indica la } \\
\text { posición de cada unidad dentro de la organización y } \\
\text { de cada persona dentro de la unidad. }\end{array}$} & Agrupación de unidades \\
\hline & & \\
\hline & & Tamaño de la unidad \\
\hline $\begin{array}{l}\text { 3. Diseño de } \\
\text { enlaces late- } \\
\text { rales }\end{array}$ & $\begin{array}{l}\text { El problema de los enlaces tiene como origen el } \\
\text { hecho de que cada grupo o unidad debe forzosa- } \\
\text { mente interactuar con las otras unidades, para ello se } \\
\text { definen los sistemas. }\end{array}$ & $\begin{array}{l}\text { Sistemas de planificación } \\
\text { y control y dispositivos de } \\
\text { enlace }\end{array}$ \\
\hline \multirow{2}{*}{$\begin{array}{l}\text { 4. Diseño del } \\
\text { sistema decisor }\end{array}$} & \multirow{2}{*}{$\begin{array}{l}\text { Para que las organizaciones cumplan sus objetivos, } \\
\text { es preciso que tomen decisiones y para ello debe dar } \\
\text { respuesta acerca de quién decide y sobre qué mate- } \\
\text { rias. }\end{array}$} & Descentralización \\
\hline & & Vertical y horizontal \\
\hline
\end{tabular}

Fuente: Adaptado de Mintzberg (1984).

Hipótesis 2 (H2). Las organizaciones que adoptan el sistema de gestión de calidad presentan una menor especialización vertical.

Hipótesis 3 (H3). Las organizaciones que adoptan el sistema de gestión de calidad presentan una mayor formalización de sus procesos. Hipótesis 4 ( 44$)$. Las organizaciones que adoptan el sistema de gestión de calidad hacen más énfasis en la preparación, dedicando más recursos a la formación de sus trabajadores. Hipótesis 5 (H5). Las organizaciones que adoptan el sistema de gestión de calidad presentan un mayor grado de adoctrinamiento de sus trabajadores.

\section{Diseño de la superestructura}

Hipótesis 6 (H6). Las organizaciones que adoptan el sistema de gestión de calidad presentan estructuras organizativas con una tendencia a disminuir el número de niveles jerárquicos.

\section{Diseño de enlaces laterales}

Hipótesis 7 ( $\mathrm{H} 7)$. Las organizaciones que adoptan el sistema de gestión de calidad utilizan más los dispositivos de enlace laterales.

\section{Diseño del sistema decisor}

Hipótesis 8 (H8). Las organizaciones que adoptan el sistema de gestión de calidad presentan un mayor grado de descentralización vertical.

\subsection{Calidad, diseño de la estructura organi- zativa e innovación}

De acuerdo con Deming (1989) se debe crear constancia en el propósito de mejora del producto y servicio. El propósito es ser competitivo, mantenerse en el negocio y proporcionar puestos de trabajo. La supervivencia a largo plazo de la empresa es objetivo prioritario, a través de medidas como la innovación y asignación de recursos a la planificación y mejora constantemente en el diseño del producto y 
servicio, en virtud de que conocer y fabricar lo que el usuario necesita, es un aspecto importante de la línea de producción.

Schumpeter (1883-1950) fue el primero en destacar la importancia de los fenómenos tecnológicos en el crecimiento económico y definió la innovación en un sentido más general que el de las innovaciones específicamente tecnológicas (Schumpeter, 1934).

A su vez, Ramis (1998) analiza diversos enfoques para diferenciar los conceptos de innovación y el término mejora continua, para lo cual cita los trabajos de algunos autores. En primer lugar, menciona a Imai (1986; citado por Ramis, 1998), el cual define innovación como el cambio del Status Quo y la mejora como una continuación con un mismo Status Quo. Por su parte, Davenport (1993; citado por Ramis, 1998), define innovación como el cambio inspirado en el pensamiento inductivo y la mejora como el cambio basado en el pensamiento deductivo. Yeo (1993; citado por Ramis, 1998), concibe la innovación como el cambio cualitativo y discontinuo por naturaleza, y la mejora como el cambio cuantitativo. Dixon (1994; citado por Ramis, 1998), define innovación como el cambio en la dirección de la trayectoria y la mejora como la continuación sobre una misma trayectoria. Por último, Stoddard (1995; citado por Ramis, 1998), concibe la innovación como el movimiento revolucionario y la mejora como el movimiento evolutivo.

La innovación continuada es factible de ser conseguida mediante cambios drásticos en los paradigmas organizacionales (Cilleruelo, 2007). La innovación es el resultado exitoso de un proceso y requiere un contexto favorecedor de una serie de características organizacionales, que constituyen un paradigma organizacional específico. Tal y como afirman Fernández, Sánchez \& Yánez (citados por Ramis, 1998) las empresas que se plantean la innovación como objetivo continuo ponen los medios adecuados para generar un paradigma organizacional favorable, lo cual implica en ocasiones, transformar valores existentes o incluso introducir otros diferentes. Cuando se hace referencia a la innovación de tipo organizacional, es necesario tener en cuenta, para su estudio en las empresas, la interrelación de diferentes componentes. Estos son elementos necesarios para aumentar la capacidad innovadora de las organizaciones (Arraut, 2010).

La relación entre los conceptos de calidad e innovación, da lugar a modelos de comportamiento empresarial en el ámbito normativo (Perdomo, 2004; citado en Arraut, 2010) y su relación con el desarrollo tecnológico y el impacto sobre la productividad y la competitividad de las empresas, el bienestar de la sociedad y el progreso de los países. La OCDE (2005), desde el punto de vista del concepto de innovación, tiene en cuenta que una empresa puede realizar múltiples innovaciones en sus métodos de trabajo, en el uso de los factores y en los procedimientos de producción para mejorar su productividad $\mathrm{y} / \mathrm{o}$ funcionamiento comercial (Arraut, 2010).

Para este apartado se planteará y contrastará una hipótesis de innovación relacionada con siete de los nueve parámetros de diseño organizativo, así:

Hipótesis 9 (H9). Las organizaciones que adoptan el sistema de gestión de calidad adaptan un diseño de la estructura que favorece las condiciones de la organización y que presenta un incremento en su potencial de innovación.

\subsection{La dulce experiencia en el valle del Rio Cauca (Colombia)}

En lo que respecta al sector azucarero en el valle del Río Cauca, en el suroccidente de Colombia, este recorre 39 municipios de los departamentos de Caldas, Cauca, Risaralda y Valle del Cauca. Es uno de 
los sectores más productivos y hace parte de los sectores agropecuario e industrial, con especialización en la producción de caña de azúcar, azúcares, mieles y alcohol (etanol). Agrupa trece ingenios azucareros, cinco plantas productoras de etanol, 2.513 unidades productoras de caña y más de cuarenta empresas de alimentos, bebidas y licores. Por estos motivos, es uno de los más desarrollados y eficientes del país.

Durante la década de los noventa el sector azucarero del valle del Rio Cauca logró consolidarse dentro del nuevo modelo de desarrollo nacional. Esto, a pesar de los problemas económicos por los que atravesaba el país, en donde el modelo entró en un periodo de revaluación que deterioró la competitividad de los bienes transables, incluyendo la azúcar; además de dificultades políticas y económicas de otros países con los que Colombia había mantenido tradicionalmente relaciones comerciales. Al ver las oportunidades de abastecer los países con los cuales Colombia empezaba a integrarse, la industria realizó grandes inversiones para modificar su política de producción y de exportaciones, incluyendo el mecanismo de franjas de precios y el arancel externo común del Grupo Andino, y la inmersión en temas como el desarrollo sostenible, el desarrollo social y el manejo ambiental.

Como resultado de estas políticas, Colombia logró establecer una industria azucarera que es punto de referencia para la industria azucarera mundial, en términos de su eficiencia y productividad, en las relaciones con sus proveedores, en su organización gremial, en materia de investigación y por los logros en materia social y ambiental. En el año 2009, en Colombia se produjeron 2,6 millones de toneladas de azúcar a partir de 23,6 millones de toneladas de caña; y 325 millones de litros de alcohol carburante, destinados a la mezcla con gasolina en una proporción E10
(10\% etanol, 90\% gasolina), de acuerdo con el mandato de oxigenación establecido por el gobierno desde noviembre de 2005. Igualmente, el valor de la producción de azúcar y alcohol fue de USD\$1.60o millones (calculado con la tasa de cambio promedio anual para el 2009), de los cuales cerca de USD $\$ 432$ millones se originaron en las exportaciones de azúcar y miel final.

En el 2009 el consumo nacional de azúcar en Colombia fue de 1,65 millones de toneladas, de los cuales el $65 \%$ se destinó a consumo directo en los hogares y un 35\% a la fabricación de productos alimenticios y bebidas para consumo humano. Además en este mismo año se exportaron un millón de toneladas de azúcar, de las cuales el $76 \%$ se dirigieron a las Islas del Caribe y Chile, Estados Unidos, Perú, México, Sri Lanka y Canadá. El resto del azúcar se exportó hacia múltiples destinos alrededor del mundo (Asocaña, 2009).

Lo anterior ha generado que el azúcar es uno de los principales productos industriales de exportación en Colombia y su industria se caracteriza por tener altos niveles de productividad con respecto a los demás competidores extranjeros, debido a que registra uno de los niveles más altos de producción de caña y de hectárea cultivada mundialmente. Según el estudio The competitive position of sugar leading industries 1997, de la firma inglesa LMC International, Colombia fue calificada dentro de la categoría de alto comportamiento técnico, la más alta categoría para un país azucarero (Asocaña, 2009).

Según Fedesarrollo (2011) los resultados de un estudio sobre el impacto socioeconómico del sector azucarero en su área de influencia y en la economía colombiana, indican que por cada empleo generado por los ingenios azucareros, se generan 28,4 empleos adicionales en otros sectores de la economía; lo que quiere decir que, gracias a la actividad 
manufacturera de los ingenios, se generan 265 mil empleos en toda la cadena de valor.

2.6 Gestión de los recursos humanos: un aliado para la implementación de la estrategia organizacional

La participación del personal es uno de los ocho principios de gestión de la calidad que pueden ser utilizados por parte de la dirección con el fin de guiar la organización hacia la una mejora en el desempeño. Dicha participación se especifica desde la norma fundamental sobre la gestión de la calidad, donde el personal, a todos los niveles, es la esencia de una organización y su total compromiso posibilita que sus habilidades sean usadas para beneficio de la organización (Icontec, 2006).

De esta manera, la alta dirección puede crear un ambiente en el que los colaboradores se involucren completamente en operar eficazmente un sistema de gestión de la calidad. Lo anterior gracias al establecimiento y mantenimiento de la política de calidad y los objetivos de la calidad, lo cual se convertirá en un aspecto importante de la cultura organizacional (Icontec, 2006). La cultura organizacional es un mecanismo estratégico para favorecer el compromiso de todas las partes que conforman la organización.

Por otra parte, es labor de la dirección de recursos humanos crear y poner en marcha diferentes estrategias que integren a los colaboradores con un comportamiento coherente a la orientación estratégica de las organizaciones y de esta manera, mantener la filosofía y sistemas de administración de la calidad que permitan el logro máximo de los objetivos y la sostenibilidad económica y financiera (Voehl, Jackson \& Ashton, 1997).

No obstante, las inversiones en capital humano a mediano plazo logran la consecución de objetivos y posiciones competitivas de mayor dificultad en el mercado (Wright \&
McMaham, 1992). Como ejemplo de ello, se encuentra la teoría de recursos y capacidades $\mathrm{y}$ en las condiciones impuestas de Barney (1991), que indican que una empresa puede obtener un valor diferenciador y una ventaja competitiva en los recursos humanos si estos le otorgan valor a los procesos de trabajo. Dicha ventaja competitiva puede darse por tres factores fundamentales: el recurso del capital físico, como es el caso de la planta física, equipos y finanzas; el recurso del capital organizacional, que implica la estructura organizacional, la planeación, coordinación y control, así como el sistema de gestión de los recursos humanos; y el capital de recursos humanos, es decir, las habilidades, inteligencia y el juicio de los colaboradores de las organizaciones (Barney \& Wright, 1998).

De esta manera se involucra la gestión de los recursos humanos como una estrategia y como un aliado para el sostenimiento de la calidad, el mejoramiento continuo, la transformación organizacional y el logro de los objetivos corporativos.

\section{Metodología}

Para este proyecto se tomó, como unidad de análisis, tres de los trece ingenios azucareros en Colombia. La escogencia de dicha muestra se basó en la facilidad en acceso al tipo de información objeto de estudio. Se espera que el estudio aporte evidencias sobre las hipótesis planteadas por medio de la elaboración y verificación del instrumento de evaluación, a través de encuestas que se aplicarán a la muestra. Finalmente, el método es cuantitativo porque contrastará las variables con un método acorde a las características de las variables.

\subsection{Método de construcción del instrumento para la recolección de la información}

Retomando la base planteada en el estudio "El sistema de gestión de calidad sistema de 
gestión de calidad y el diseño de la estructura organizativa: un estudio empírico" de Bayo \& Merino (2003); así como el marco conceptual definido por Mintzberg (1984) referente al a los parámetros de diseño organizativo; se realizó el proceso de construcción del instrumento para la recolección de la información, teniendo en cuenta las diferentes hipótesis postuladas en la investigación de Bayo \& Merino. Así mismo, se tomaron en cuenta los alcances y definiciones de cada uno de los parámetros de diseño de las hipótesis con el fin de construir los diferentes ítems o frases que fueron cuidadosamente seleccionadas, de forma que construyeran un criterio válido, fiable y preciso para medir de alguna forma lo planteado en el proyecto (Hernández, Fernández \& Baptista, 1998).

Por otra parte, dichas frases, correspondientes a los diferentes parámetros de diseño de cada uno de los objetos de estudio (del diseño del puesto, el diseño de la superestructura, el diseño de enlaces laterales y el diseño del sistema decisor); fueron construidas bajo la modalidad de la escala Likert, la cual es una escala ordinal que permite identificar resultados más favorables al comparar las muestras de la población en estudio: ingenios del sector azucarero (Hernández et al., 1998).

\subsection{Proceso para la validación del instrumen- to y para la recolección de datos}

La validación del presente instrumento se llevó a cabo mediante dos procedimientos complementarios: la evaluación cualitativa y la determinación de la consistencia interna. El primer procedimiento se llevó a cabo a través de la consulta a expertos: dos docentes, de pregrado y postgrado, especialistas en el área de diseño organizacional que juzgaron críticamente el instrumento y sugirieron hacer los ajustes necesarios. Posteriormente, se aplicó la prueba piloto a tres empresas de otros sectores y a colaboradores de distintos niveles jerárquicos; finalmente se conformó una muestra de treinta encuestados y se analizaron los resultados de la prueba piloto en compañía de un experto en estadística.

\subsection{Proceso para la recolección de informa- ción}

En referencia a la aplicación del instrumento, éste se aplicó directamente, tomando muestras de tres ingenios conformadas por directivos, coordinadores y personal operativo de manera aleatoria. Los dos primeros ingenios cuentan con un sistema de gestión de la calidad ya impreso en la política y en el quehacer organizacional en todos sus niveles, mientras que el tercero lo posee en la calidad del producto terminado más no en todos los aspectos correspondientes al funcionamiento de la organización. De esta manera, se explican a continuación las muestras correspondientes a los ingenios que hicieron parte del estudio:

- Ingenio $1\left(\mu_{1}\right): 25$ encuestas aplicadas.

- Ingenio $2\left(\mu_{2}\right): 21$ encuestas aplicadas.

- Ingenio $3\left(\mu_{3}\right): 23$ encuestas aplicadas.

Lo anterior permitió recoger información valiosa de tres ingenios ubicados en el Valle del Cauca con el fin de analizar y realizar comparaciones entre las muestras. Posteriormente, se realizó el análisis de la información utilizando diferentes pruebas de hipótesis para cada una de las muestras.

\section{Resultados}

En el análisis de resultados se parte del supuesto que las variables de interés poseen varianzas iguales y siguen una distribución normal, dado que las muestras se consideran pequeñas (tamaño muestral inferior a treinta observaciones). Este supuesto se establece con el fin de realizar el correspondiente análisis de Kolgomorov-Smirnoff, utilizando la distribución $t$, y para determinar los grados de libertad (Levin \& Rubin, 2010). 
Sin embargo, en relación al análisis de normalidad de las variables efectuado por la prueba Kolgomorov-Smirnoff, se encontró que los datos correspondientes a las hipótesis H1, H2, H3 Y H4 no tenían una distribución normal. Por este motivo, se realizó la prueba de Kruskal Wallis para pruebas no paramétricas; que permitió elaborar un análisis de resultados acorde a las variables y los datos.

Así mismo, se utilizó el programa estadístico spss V.19 con una prueba de ANova para las tres muestras, según el método de comparación múltiple de Tukey, con el objetivo de contrastar entre las dos pruebas, el análisis de la información y las hipótesis del presente estudio. Se asume entonces que las tres muestras son iguales. A continuación se presentan los resultados obtenidos en las diferentes hipótesis formuladas, las cuales están relacionadas directamente con los objetivos.

Hipótesis 1 (H1). Las organizaciones que adoptan el sistema de gestión de calidad presentan una menor especialización horizontal, es decir, $H_{0}: \mu_{1}=\mu_{2}=\mu_{3} ; H_{a}:$ Algún $\mu_{i}$ es diferente.

Los resultados se muestran en la Tabla 2 y en la Tabla 3, donde teniendo en cuenta el $\alpha=0,05$ y el $p$-valor $=0,072$, no se encontraron diferencias estadísticamente significativas entre las muestras.

Igualmente se analizó la especialización horizontal, la cual es la cantidad de tareas que son asignadas a un puesto de trabajo y el alacance de las mismas, es decir, entre más especializado horizontalmente un cargo, mayor especialización en las funciones del trabajador. Para el caso de los resultados obtenidos, se puede interpretar que dicho aspecto se presenta en los tres ingenios evaluados, es decir, existe una especialización horizontal, sin embargo, se percibe que si un ingenio no posee un sistema de gestión de calidad en todos los niveles de la organización, se podría presentar mayor satisfacción de personal, rotación del mismo entre diferentes áreas y mayor esfuerzo por la formación de los colaboradores, lo cual podría favorecer la disminución de la especialización horizontal.

Hipótesis 2 (H2). Las organizaciones que adoptan el sistema de gestión de calidad presentan una menor especialización vertical, es decir, $H_{0}: \mu_{1}=\mu_{2}=\mu_{3} ; H_{a}$ : Algún $\mu_{i}$ es diferente.

Tabla 2. Prueba de ANOva para hipótesis 1

\begin{tabular}{lccccc}
\hline H1 & Suma de cuadrados & gl & Media cuadrática & F & Sig. \\
\hline Inter-grupos & 11,516 & 2 & 5,758 & 2,733 & 0,072 \\
Intra-grupos & 138,035 & 66 & 2,107 & & \\
Total & 150,551 & 68 & & & \\
\hline
\end{tabular}

Tabla 3. Método de comparación múltiple HSD de Tukey para hipótesis 1

\begin{tabular}{lcc}
\hline & $\mathrm{N}$ & Subconjunto para $\alpha=0,05$ \\
\cline { 3 - 3 } Ingenio & 25 & 1 \\
\hline Ingenio 1 & 23 & 6,52 \\
Ingenio 2 & 21 & 6,57 \\
Ingenio 3 & & 7,43 \\
Sig. & & 0,094 \\
\hline
\end{tabular}


Los resultados se muestran en la Tabla 4 y en la Tabla 5, donde teniendo en cuenta el $\alpha=0,05$ y el $p$-valor $=0,000$; se puede inferir que existen diferencias estadísticamente significativas en alguno de los ingenios. El análisis de comparaciones múltiples entre las muestras con el fin de identificar cuáles son homogéneos.

Al compararse las muestras entre sí, se puede inferir que no existe diferencia estadística significativa entre el ingenio 2 y el ingenio 3 , de los cuales, el primero tiene el sistema de gestión de calidad en todos los niveles, mientras el segundo únicamente en el producto terminado.

Para el caso de la especialización vertical, se podría suponer que en el ingenio1, siendo diferente significativamente con el ingenio 2 y 3 ; tendría un mayor control por parte de los directivos frente a las funciones de sus colaboradores; lo que permitiría suponer una reducción en la participación de éstos en la toma de decisiones y un menor incremento en sus responsabilidades, lo cual puede ser común en los puestos operativos.

Hipótesis $3(\mathrm{H} 3)$. Las organizaciones que adoptan el sistema de gestión de calidad presentan una mayor formalización de sus procesos, es decir, $H_{0}: \mu_{1}=\mu_{2}=\mu_{3} ; H_{a}:$ Algún $\mu_{i}$ es diferente.

Los resultados se muestran en la Tabla 6 y en la Tabla 7 , donde teniendo en cuenta el $\alpha=0,05$ y el $p$-valor $=0,419$; se puede inferir que no se encontraron diferencias significativas entre las tres muestras, lo que sugiere que en los tres ingenios evaluados, existe suficiente formalización a los procesos, per-

Tabla 4. Prueba de ANOva para hipótesis 2

\begin{tabular}{lccccc}
\hline H2 & Suma de cuadrados & gl & Media cuadrática & F & Sig. \\
\hline Inter-grupos & 25,895 & 2 & 12,947 & 19,529 & 0,000 \\
Intra-grupos & 43,758 & 66 & 0,663 & & \\
Total & 69,652 & 68 & & & \\
\hline
\end{tabular}

Tabla 5. Método de comparación múltiple HsD de Tukey hipótesis 2

\begin{tabular}{lccc}
\hline & & \multicolumn{2}{c}{ Subconjunto para $\alpha=0,05$} \\
\cline { 3 - 4 } Ingenio & $\mathrm{N}$ & 1 & 2 \\
\hline Ingenio 1 & 25 & 2,84 & \\
Ingenio 2 & 23 & & 4,09 \\
Ingenio 3 & 21 & & 4,14 \\
Sig. & & 1,000 & 0,971 \\
\hline
\end{tabular}

Tabla 6. Prueba de ANOva para hipótesis 3

\begin{tabular}{lccccc}
\hline H3 & Suma de cuadrados & gl & Media cuadrática & F & Sig. \\
\hline Inter-grupos & 2,139 & 2 & 1,070 & \multirow{2}{*}{0,881} & 0,419 \\
Intra-grupos & 80,151 & 66 & 1,214 & & \\
Total & 82,290 & 68 & & & \\
\hline
\end{tabular}


mitiendo que los colaboradores identifiquen los alcances de sus puestos de trabajo, mejoren significativamente su productividad, identifiquen sus funciones y el aporte que desde ahí realizan para la consecución de los objetivos estratégicos de las organizaciones (ver Tabla 7).

Hipótesis 4 ( $\mathrm{H} 4)$. Las organizaciones que adoptan el sistema de gestión de calidad hacen más énfasis en la preparación dedicando más recursos hacia la formación de sus trabajadores, es decir, $H_{o}: \mu_{1}=\mu_{2}=\mu_{3} ; H_{a}$ : Algún $\mu_{i}$ es diferente.

Los resultados se muestran en la Tabla $8 \mathrm{y}$ en la Tabla 9. Para el caso de la hipótesis 4, se puede inferir que no se encontraron diferencias significativas entre aquellos que poseen sistema de gestión de calidad y la muestra 3 (ver Tabla 8) de acuerdo a el p-valor $=0,097$. Sin embargo, se evidencia que los resultados del ingenio 2 y 3 son similares a los resultados del ingenio 1 y 2 , lo cual era un resultado esperado con esta prueba de hipótesis. Lo anterior supone que, en los ingenios similares, se auspicia la destinación de mayores recursos a la formación constante de los colaboradores con el fin que estos mejoren sus habilidades, competencias y niveles de experticia en las labores que ejecutan en sus puestos de trabajo (ver Tabla 9).

Hipótesis 5 (H5). Las organizaciones que adoptan el sistema de gestión de calidad presentan un mayor grado de adoctrinamiento de sus trabajadores, es decir, $H_{0}: \mu_{1}=\mu_{2}=\mu_{3}$; $H_{a}$ : Algún $\mu_{i}$ es diferente.

Tabla 7. Método de comparación múltiple HSD de Tukey hipótesis 3

\begin{tabular}{lcc}
\hline \multirow{2}{*}{ Ingenio } & $\mathrm{N}$ & Subconjunto para $\alpha=0,05$ \\
\cline { 3 - 3 } & & 1 \\
\hline Ingenio 1 & 25 & 7,08 \\
Ingenio 2 & 21 & 7,14 \\
Ingenio 3 & 23 & 7,48 \\
Sig. & & 0,444 \\
\hline
\end{tabular}

Tabla 8. Prueba de anova para hipótesis 4

\begin{tabular}{lccccc}
\hline H4 & Suma de cuadrados & gl & Media cuadrática & $\mathrm{F}$ & Sig. \\
\hline Inter-grupos & 22,153 & 2 & 11,077 & 2,637 & 0,079 \\
Intra-grupos & 277,180 & 66 & 4,200 & & \\
Total & 299,333 & 68 & & & \\
\hline
\end{tabular}

Tabla 9. Método de comparación múltiple HSD de Tukey hipótesis 4

\begin{tabular}{lcc}
\hline \multirow{2}{*}{ Ingenio } & $\mathrm{N}$ & Subconjunto para $\alpha=0,05$ \\
\cline { 3 - 3 } & & 1 \\
\hline Ingenio 1 & 23 & 5,87 \\
Ingenio 2 & 25 & 7,00 \\
Ingenio 3 & 21 & 7,14 \\
Sig. & & 0,097 \\
\hline
\end{tabular}


Los resultados se muestran en la Tabla $10 \mathrm{y}$ en la Tabla 11. En relación al adoctrinamiento, no se encontraron diferencias estadísticas significativas entre los tres ingenios, es decir, independientemente de la implementación de un sistema de gestión de calidad, dichas organizaciones favorecen la integración de los colaboradores a la misma e invierten recursos para incrementar el sentido de pertenencia, así como la interiorización de la política y estrategia de la organización. Lo anterior podría ser liderado en el caso de los tres ingenios por los directivos, los cuales se encargarían de transmitirlo a sus equipos de trabajo, reconociendo además la importancia del recurso humano.
Hipótesis 6 (н6). Las organizaciones que adoptan el sistema de gestión de calidad presentan estructuras organizativas con una tendencia a disminuir el número de niveles jerárquicos, es decir, $H_{0}: \mu_{1}=\mu_{2}=\mu_{3} ; H_{a}:$ Algún $\mu_{i}$ es diferente.

Los resultados se muestran en la Tabla 12 y en la Tabla 13. En relación a la hipótesis 6 , se puede inferir que existen diferencias estadísticamente significativas en alguno de los ingenios, particularmente en el ingenio 3, el cual muestra un valor de significancia mayor; sin embargo, el ingenio 1 y el ingenio 2 poseen similitud entre sí, lo cual podría implicar la existencia de mayores niveles jerárquicos, generando así una organización centralizada donde predomina la jerarquía de la autoridad.

Tabla 10. Prueba de ANova para hipótesis 5

\begin{tabular}{lccccc}
\hline $\mathrm{H}_{5}$ & Suma de cuadrados & gl & Media cuadrática & F & Sig. \\
\hline Inter-grupos & 0,859 & 2 & 0,429 & 0,106 & 0,899 \\
Intra-grupos & 266,098 & 66 & 4,032 & \\
Total & 266,957 & 68 & & \\
\hline
\end{tabular}

Tabla 11. Método de comparación múltiple HSD de Tukey hipótesis 5

\begin{tabular}{lcc}
\hline Ingenio & $\mathrm{N}$ & Subconjunto para $\alpha=0,05$ \\
\cline { 3 - 3 } & & 1 \\
\hline Ingenio 1 & 23 & 12,43 \\
Ingenio 2 & 25 & 12,56 \\
Ingenio 3 & 21 & 12,71 \\
Sig. & & 0,885 \\
\hline
\end{tabular}

Tabla 12. Prueba de ANOva para hipótesis 6

\begin{tabular}{lccccc}
\hline H6 & Suma de cuadrados & gl & Media cuadrática & F & Sig. \\
\hline Inter-grupos & 113,607 & 2 & 56,804 & 8,871 & 0,000 \\
Intra-grupos & 422,596 & 66 & 6,403 & & \\
Total & 536,203 & 68 & & & \\
\hline
\end{tabular}


Hipótesis 7 (H7). Las organizaciones que adoptan el sistema de gestión de calidad utilizan más de los dispositivos de enlace laterales, es decir, $H_{0}: \mu_{1}=\mu_{2}=\mu_{3} ; H_{a}:$ Algún $\mu_{i}$ es diferente.

Los resultados se muestran en la Tabla 14. Teniendo en cuenta el $\alpha=0,05$ y un $p$-valor $=$ o,049; se puede inferir que existen diferencias significativas entre las muestras $y$, para este caso en referencia a los tres ingenios, es de destacar que en los mismos se realizan los diferentes grupos de mejora continua teniendo presente la relación entre calidad y grupos de trabajo, siendo lo anterior una oportunidad para la implantación de nuevas tecnologías que permitan ser mucho más eficientes y competitivos. Por otra parte, se evidencia que el ingenio 3 parece tener una implementación más favorable en el tema de los enlaces laterales y equipos de trabajo (ver Tabla 15).

Hipótesis 8 (H8). Las organizaciones que adoptan el sistema de gestión de calidad presentan un mayor grado de descentralización vertical, es decir, $H_{0}: \mu_{1}=\mu_{2}=\mu_{3} ; H_{a}:$ Algún $\mu_{i}$ es diferente.

Los resultados se muestran en la Tabla 16 y 17 , donde se puede inferir de acuerdo a los resultados obtenidos, se puede inferir que

Tabla 13. Método de comparación múltiple HsD de Tukey hipótesis 6

\begin{tabular}{lccc}
\hline \multirow{2}{*}{ Ingenio } & $\mathrm{N}$ & \multicolumn{2}{c}{ Subconjunto para $\alpha=0,05$} \\
\cline { 3 - 4 } & & 1 & 2 \\
\hline Ingenio 1 & 25 & 16,04 & \\
Ingenio 2 & 21 & 17,10 & 19,09 \\
Ingenio 3 & 23 & & 1,000 \\
Sig. & & 0,341 & \\
\hline
\end{tabular}

Tabla 14. Prueba de ANOva para hipótesis 7

\begin{tabular}{lccccc}
\hline H7 & Suma de cuadrados & gl & Media cuadrática & F & Sig. \\
\hline Inter-grupos & 23,921 & 2 & 11,961 & 3,160 & 0,049 \\
Intra-grupos & 249,847 & 66 & 3,786 & & \\
Total & 273,768 & 68 & & & \\
\hline
\end{tabular}

Tabla 15. Método de comparación múltiple HsD de Tukey hipótesis 7

\begin{tabular}{lccc}
\hline \multirow{2}{*}{ Ingenio } & $\mathrm{N}$ & \multicolumn{2}{c}{ Subconjunto para $\alpha=0,05$} \\
\cline { 3 - 4 } & & 1 & 2 \\
\hline Ingenio 1 & 21 & 15,48 & \\
Ingenio 2 & 25 & 15,80 & 15,80 \\
Ingenio 3 & 23 & & 16,87 \\
Sig. & & 0,840 & 0,159 \\
\hline
\end{tabular}


existen diferencias estadísticamente significativas entre las muestras de los ingenios en relación a la hipótesis. Por otra parte, es concluyente la similitud de resultados de $\mu_{2}$ con $\mu_{1}$ y a su vez $\mu_{3}$ con $\mu_{2}$. Lo anterior podría implicar que el ingenio 2 y el 3 tendrían una mayor descentralización en la toma de decisiones, suponiendo que se trasfiere más poder para la toma de decisiones a los niveles más bajos de la estructura organizacional y se promueve en mayor medida la descentralización vertical (ver Tabla 17).

Hipótesis 9 (н9). Las organizaciones que adoptan el sistema de gestión de calidad adaptan un diseño de la estructura que favorece las condiciones de la organización y que presenta un incremento en su potencial de innovación, es decir, $H_{o}: \mu_{1}=\mu_{2}=\mu_{3} ; H_{a}$ : Algún $\mu_{i}$ es diferente.

Los resultados se muestran en la Tabla 18 donde teniendo en cuenta el $\alpha=0,05$ y un p-valor $=0,268$; esta prueba de hipótesis no se rechazó, por lo tanto se puede afirmar, con un nivel de confianza del $95 \%$, que no existe suficiente evidencia estadística para afirmar que existe una diferencia significativa estadísticamente en la innovación de los tres ingenios. Como se puede apreciar en la Tabla 19, hay factores que no evidencian el incremento del potencial de innovación.

\section{Conclusiones}

Este estudio representa una contribución al conocimiento sobre la relación entre el

Tabla 16. Prueba de ANOva para hipótesis 8

\begin{tabular}{lccccc}
\hline H8 & Suma de cuadrados & gl & Media cuadrática & F & Sig. \\
\hline Inter-grupos & 43,597 & 2 & 21,798 & 4,812 & 0,011 \\
Intra-grupos & 298,954 & 66 & 4,530 & & \\
Total & 342,551 & 68 & & & \\
\hline
\end{tabular}

Tabla 17. Método de comparación múltiple HSD de Tukey hipótesis 8

\begin{tabular}{lccc}
\hline \multirow{2}{*}{ Ingenio } & $\mathrm{N}$ & \multicolumn{2}{c}{ Subconjunto para alfa $=0,05$} \\
\cline { 3 - 4 } & 25 & 12,92 & 2 \\
\hline Ingenio 1 & 21 & 13,90 & 13,90 \\
Ingenio 2 & & 14,83 \\
Ingenio 3 & 23 & 0,268 & 0,315 \\
\hline Sig. & & & \\
\hline
\end{tabular}

Tabla 18. Prueba de ANOva para hipótesis 9. ANOvA - relación innovación - parámetros de diseño organizativo

\begin{tabular}{lccccc}
\hline H9 & Suma de cuadrados & gl & Media cuadrática & F & Sig. \\
\hline Inter-grupos & 97,656 & 2 & 48,828 & 1,345 & 0,268 \\
Intra-grupos & 2396,170 & 66 & 36,306 & & \\
Total & 2493,826 & 68 & & & \\
\hline
\end{tabular}


Tabla 19. Estadística descriptiva para cada uno de los factores analizados en los tres ingenios

\begin{tabular}{|c|c|c|c|c|c|c|}
\hline Ingenio & & $\mathrm{N}$ & Mínimo & Máximo & Media & Desv. Est. \\
\hline \multirow[t]{19}{*}{1} & $\mathrm{~V} 1$ & 25 & 2 & 5 & 4,28 & 0,678 \\
\hline & $\mathrm{V} 2$ & 25 & 2 & 5 & 4,04 & 0,790 \\
\hline & V3 & 25 & 3 & 5 & 4,24 & 0,597 \\
\hline & $\mathrm{V} 4$ & 25 & 3 & 5 & 4,24 & 0,523 \\
\hline & V5 & 25 & 1 & 5 & 3,56 & 0,917 \\
\hline & V6 & 25 & 1 & 5 & 3,44 & 0,870 \\
\hline & V7 & 25 & 3 & 5 & 4,08 & 0,812 \\
\hline & V8 & 25 & 1 & 5 & 2,76 & 1,128 \\
\hline & V9 & 25 & 2 & 5 & 3,44 & 0,961 \\
\hline & V10 & 25 & 4 & 5 & 4,28 & 0,458 \\
\hline & V11 & 25 & 2 & 5 & 3,48 & 0,823 \\
\hline & $\mathrm{V} 12$ & 25 & 2 & 5 & 3,80 & 0,816 \\
\hline & V13 & 25 & 2 & 5 & 4,24 & 0,879 \\
\hline & V14 & 25 & 2 & 5 & 3,72 & 0,936 \\
\hline & V15 & 25 & 2 & 5 & 3,52 & 0,823 \\
\hline & V16 & 25 & 1 & 5 & 2,36 & 0,907 \\
\hline & V17 & 25 & 2 & 5 & 3,32 & 0,945 \\
\hline & Tpys; & 25 & 52 & 5 & 62,80 & 6,745 \\
\hline & N Valido (list wise) & 25 & & & - & - \\
\hline \multirow[t]{8}{*}{2} & $\mathrm{~V} 1$ & 21 & 3 & 5 & 4,29 & 0,561 \\
\hline & $\mathrm{V} 2$ & 21 & 3 & 5 & 4,19 & 0,680 \\
\hline & V3 & 21 & 3 & 5 & 4,24 & 0,700 \\
\hline & V4 & 21 & 2 & 5 & 4,38 & 0,740 \\
\hline & V5 & 21 & 1 & 5 & 3,57 & 1,165 \\
\hline & V6 & 21 & 2 & 5 & 3,57 & 1,028 \\
\hline & V7 & 21 & 2 & 5 & 4,00 & 1,095 \\
\hline & V8 & 21 & 1 & 5 & 3,00 & 0,949 \\
\hline
\end{tabular}

sistema de gestión de calidad y el diseño organizativo, y a su vez con la innovación, en las empresas vinculadas al sector del azúcar en Colombia. Se considera que las conclusiones del estudio tienen validez, bajo el sustento de un riguroso análisis estadístico de la información, al adecuado planteamiento de hipótesis y a la estructuración de la herramienta de medición acorde con los objetivos de la investigación.
Es necesario destacar que en el sector azucarero colombiano no se puede afirmar, hasta el momento, que la implementación de un sistema de gestión de calidad necesariamente haya ocasionado cambios en el diseño organizativo, pues como se pudo observar con algunas de las hipótesis estudiadas, existieron grandes similitudes entre el ingenio que no cuenta con el sistema de gestión de calidad y el ingenio 2. Sin 
embargo, dichos cambios organizacionales podrían ser fomentados por la necesidad de adaptarse al cambio socioeconómico del sector y de ésta manera mantener su crecimiento y aporte al PIB. Por otra parte, no se evidenció que exista una diferencia significativa estadísticamente en la innovación de los tres ingenios.

Igualmente, se debe tener en cuenta que el sector azucarero colombiano posee una historia particular enmarcada en la posición estratégica de cada uno de los ingenios en el mercado y a su respectiva transformación hacia la nueva creación de líneas de negocio (alcohol carburante, melazas, insumos a la industria papelera, generación de energía, entre otros). Lo anterior ha permitido la consolidación de estructuras organizacionales de acuerdo a su modelo de negocio, teniendo en cuenta el trabajo de las áreas de recursos humanos para el apoyo y mayor aprovechamiento de los colaboradores.

Es importante destacar en este estudio los resultados obtenidos en relación a las hipótesis de formación, formalización y adoctrinamiento, pues se percibe que los tres ingenios destinan recursos humanos, ambientales y económicos con el fin de lograr que sus colaboradores tengan la formación necesaria para realizar sus funciones e identifiquen cuál es el aporte que hacen al logro de los objetivos estratégicos de la organización. Así mismo, se considera tener en cuenta para futuras investigaciones en el tema, otras variables que permitan enriquecer la labor investigativa y comprobar así los planteamientos con mayor detalle y extender el estudio a otros contextos organizacionales.

En definitiva, es de suponer que el estudio realiza un aporte significativo a la investigación referente a los sistemas de gestión de calidad, al desarrollo de estructura organizativa, al diseño de puestos de trabajo y al sector azucarero colombiano. Este último se encuentra en una transición hacia la menor dependencia del azúcar como único producto y a la adaptación a los diferentes cambios del entorno nacional e internacional que requieren una constante innovación de su modelo de negocio. De esta manera, este estudio haría una contribución a los directivos de los ingenios pues se evidencian algunos aspectos que podrían ser una oportunidad de mejora, como es el caso de la toma de decisiones, la jerarquía y la creación de mayores equipos de trabajo, sin dejar a un lado las fortalezas ya adquiridas que han permitido el crecimiento y sostenibilidad a largo plazo.

Dada la importancia que tiene el sector azucarero para la economía colombiana, se recomienda que para futuras investigaciones se amplíe este estudio descriptivo a más ingenios, pues solo se estudiaron tres de las trece organizaciones en esta industria en Colombia. Por otro lado, sería interesante involucrar las gerencias de gestión humana y planeación para que el correspondiente análisis de la información pueda contar con insumos que validen o hagan más explícitos los resultados obtenidos, como es el caso de la cultura organizacional y objetivos estratégicos. Así mismo, sería de gran impacto que organizaciones como Cenicaña y Asocaña, participaran de manera activa en la investigación de temas que hacen parte de las empresas como es el caso de éste; pues sería un avance que permitiría cumplir su visión estratégica del sector en el año 2030, según la cual el sector azucarero colombiano será el líder en la agroindustria internacional por su competitividad, sostenibilidad y generación de bienestar (Asocaña, 2011).

Finalmente, sería de gran importancia que las organizaciones del sector se interesen por incluir en las diferentes mediciones anuales algunas variables del presente es- 
tudio, ya que podrían aportar información valiosa para el análisis de otras herramientas como es el caso de las evaluaciones de clima organizacional.

\section{Referencias bibliográficas}

Andersen, J.A. (2002). Organizational design: two lessons to learn before reorganizing. International Journal of Organization Theory and Behavior, 5(3-4), 343-358.

Arraut, L.C. (2010). Quality management as an organizational innovation for the productivity of the company. Revista EAN, 69, 20-41.

Asocaña. (2009). Informe anual (20082009): Sector azucarero. Disponible en http://www.asocana.org/publico/info. aspx?Cid=8

Asocaña. (2011). Informe anual (20102011): Sector azucarero. Disponible en http://www.asocana.org/publico/info. aspx?Cid=8

Barney, J.B. (1991). Firm resources and sustained competitive advantage. Journal of Management, 17(1), 99-120.

Barney, J.B. \& Wright, P.M. (1998). On becoming a strategic partner: The role of human resources in gaining competitive advantage. Human Resource Management, $37(1), 31-46$.

Bayo, A. \& Merino, J. (2003). La gestión de la calidad total y el diseño de la estructura organizativa, un estudio empírico. Cuadernos de economía y dirección de la empresa, 15, 118-135.

Beer, M. \& Walton, A.E. (1987). Organization Change and Development. Annual Review of Psychology, 38, 339-367.

Blocher, E.J., Stout, D.E., Cokins, G. \& Chen, K. (2008). Administración de costos: Un enfoque estratégico ( ${ }^{\mathrm{a}}$ ed.). México DF: McGraw-Hill Interamericana de México.
Chandler, A.D. (1962). Strategy and structure: Chapters in the history of the industrial enterprise. Cambridge, MA: MIT Press. Cilleruelo, E. (2007). Compendio de definiciones del concepto innovación realizadas por autores relevantes: Diseño híbrido actualizado del concepto. Revista Dirección y Organización D \& O, 34, 91-98.

Daft, R.L. (2007). Teoría y diseño organizacional. México: Cengage Learning.

Deming, E. (1989). Calidad, productividad y competitividad. La salida de la crisis. Madrid: Díaz de Santos.

Fedesarrollo. (2011). Cuadernos de Fedesarrollo, 31. Disponible en http:// www.fedesarrollo.org.co/wp-content/ uploads/2011/08/Cuaderno-No-31.pdf

Grupo Manuelita. (2011). Historia. Disponible en http://www.manuelita.com/ index.php? $\mathrm{p}=$ quienessomos/historia

Hernández, R., Fernández, C. \& Baptista, P. (1998). Metodología de la investigación. México: McGraw-Hill.

Icontec. (2006). Normas fundamentales sobre la gestión de la calidad y documentos de orientación para su aplicación. Bogotá: Autores.

Jones, G.R. \& Ruíz, C. (2008). Book and Words Solutions; Guerrero, P. M.; Teoría organizacional: Diseño y cambio en las organizaciones ( $5^{\mathrm{a}} \mathrm{ed}$.). México: Pearson. Levin, R. \& Rubin, D. (2010). Estadística para administración y economía ( $7^{\mathrm{a}} \mathrm{ed}$.). México: Pearson.

Menguzzato, M. \& Renau, J.J. (1992). La dirección estratégica de la empresa: un enfoque innovador del management. Barcelona: Ariel.

Mintzberg, H. (1984). La estructuración de las organizaciones. Barcelona: Ariel.

Mintzberg, H. \& Lampel, J. (1999). Reflecting on the strategy process. Sloan Management Review, 40(3), 21. 
Mintzberg, H., Quinn, J. \& Voyer J. (1997). El proceso estratégico: Conceptos, contextos y casos. México: Prentice-Hall.

OCDE. (2005). Oslo Manual: Guidelines for Collecting and Interpreting Innovation (3a ed.). Paris: Autores.

Pinto, J.J., Soto, N.Y, Gutiérrez, A. \& Castillo, L.J. (2003). Ajuste, estructura y ambiente como factores claves en la teoría de contingencias. Estudios gerenciales, 88, 67-86. Disponible en http://www.icesi.edu.co/ revistas/index.php/estudios_gerenciales/ article/view/115/113

Ramis, J. (1998). A Profound Study of Change in Process Innovation Projects. Barcelona: IESE.

Rico, R. \& Fernández, M. (2002). Diseño de organizaciones como proceso simbólico. Psicothema, 14(2), 415-425.

Ríos, J. (2000). Revisión de la jungla de la teoría administrativa. Revista contaduría y administración, 199, 55-74.

Schumpeter, J.A. (1934). The Theory of Economic Development. New York, NY: McGraw-Hill.

Van de Ven, A.H. \& Drazin, R. (1985). Alternative forms of fit in contingency theory. Administrative science quarterly, 3o(4), 514-539.

Voehl, F., Jackson, P. \& Ashton, D. (1997). Iso 9000. Guía de instrumentación para pequeñas y medianas empresas. Mexico: McGraw-Hill.

Wright, P.M. \& MacMahan, G.C. (1992). Theoretical perspectives for strategic human resources management. Journal of Management, 18(2), 295-320. 